Research Paper

\title{
Analysis of inflammatory parameters and disease severity for 88 hospitalized COVID-19 patients in Wuhan, China
}

\author{
Xia $\mathrm{Xu}^{1}$, Mu-Qing $\mathrm{Yu}^{2}$, Qian Shen ${ }^{3}$, Lian-Zhong Wang, ${ }^{4}$ Rong-Di Yan ${ }^{4}$, Meng-Yu Zhang ${ }^{5}$, Jian-Yu Liu ${ }^{5}$ and \\ Yi-Qing $\mathrm{Qu}^{6}$ \\ 1. Department of Geriatric Medicine, Qilu Hospital of Shandong University \\ 2. Department of Pulmonary and Critical Care Medicine, Tongji Hospital, Tongji Medical College, Huazhong University of Science and Technology. \\ 3. Department of Oncology, Tongii Hospital, Tongii Medical College, Huazhong University of Science and Technology. \\ 4. Department of Pulmonary and Critical Care Medicine, The Second Affiliated Hospital of Shandong University of TCM. \\ 5. Department of Pulmonary and Critical Care Medicine, Qilu Hospital, Cheeloo College of Medicine, Shandong University, Jinan 250012, China. \\ 6. Department of Pulmonary and Critical Care Medicine, Qilu Hospital of Shandong University.
}

$\triangle$ Corresponding author: Prof. Yi-Qing Qu, MD, PhD, Department of Pulmonary and Critical Care Medicine, Qilu Hospital of Shandong University, Wenhuaxi Road 107\#, Jinan 250012, China. Tel: +86 5318216 9335; Fax: +86 5318296 7544; E-mail: quyiqing@sdu.edu.cn.

(C) The author(s). This is an open access article distributed under the terms of the Creative Commons Attribution License (https://creativecommons.org/licenses/by/4.0/). See http://ivyspring.com/terms for full terms and conditions.

Received: 2020.05.08; Accepted: 2020.07.18; Published: 2020.07.25

\begin{abstract}
Background and aim: The outbreak of coronavirus disease 2019 (COVID-19) is quickly turning into a pandemic. We aimed to further clarify the clinical characteristics and the relationship between these features and disease severity.

Methods: In this retrospective single-center study, demographic, clinical and laboratory data were collected and analyzed among moderate, severe and critically ill group patients.

Results: 88 hospitalization patients confirmed COVID-19 were enrolled in this study. The average age of the patients was 57.11 years (SD, \pm 15.39$)$. Of these 88 patients, the median body mass index (BMI) was 24.03 (IQR, 21.64-26.61; range 15.05-32.39), the median duration from disease onset to hospital admission were 11 days (IQR, 6.50-14.50). 46.59\% patients had one or more comorbidities, with hypertension being the most common $(26.14 \%)$, followed by diabetes mellitus $(12.50 \%)$ and coronary atherosclerotic heart disease (CAD) (7.95\%). Common symptoms at onset of disease were fever (71.59\%), cough (59.09\%), dyspnea (38.64\%) and fatigue (29.55\%). 88 patients were divided into moderate (47 [53.41\%]), severe (32 [36.36\%]) and critically ill (9 [10.23\%]) groups. Compared with severe and moderate patients, lymphocytopenia occurred in $85.71 \%$ critically ill patients, and serum IL-2R, IL-6, IL-8, TNF-a, LDH, and cTnl were also increased in $71.42 \%, 83.33 \%, 57.14 \%, 71.43 \%, 100 \%$ and $42.86 \%$ in critically ill patients. Through our analysis, the age, comorbidities, lymphocyte count, eosinophil count, ferritin, CRP, LDH, PT and inflammatory cytokines were statistically significant along with the disease severity.

Conclusion: We found some clinical characteristic and inflammatory cytokines could reveal the severity of COVID-19 during the outbreak phage. Our research could assist the clinicians recognize severe and critically ill patients timely and focus on the expectant treatment for each patient.
\end{abstract}

Key words: COVID-19; outbreak phage; clinical characteristics; inflammatory cytokines; disease severity

\section{Introduction}

In December 2019, Coronavirus Disease 2019 (COVID-19), a fatal zoonotic disease, occurred in Wuhan, Hubei Province, China. The disease which was caused by 2019 novel coronavirus (2019-nCoV) has rapidly spread. Up to March 8, 2020, a total of 80735 COVID-19 cases in China had been confirmed. The pathogen could cause severe respiratory syndrome, including fever, dyspnea, and cough [1-7], 
along with other systematic damage like acute cardiac or kidney injury [8]. The transmission from person to person, caused by a propagated source, was the primary liability to the rapidly outbreak in China even the worldwide [9].

At the early phase of COVID-19, researches indicated that COVID-19 was similar with severe acute respiratory syndrome (SARS) and Middle East respiratory syndrome (MERS) in epidemiology, clinical features, and diagnosis [6,7]. Primitive case reports revealed the common symptoms were fever, cough, and myalgia or fatigue, which were similar with normal influenza [10]. They estimated the severity of COVID-19 by whether patients living in intensive care unit (ICU), which may neglect some severe patients, and the relationship between disease characteristic, several inflammatory cytokines and disease severity was still not quite clear.

As time went by, COVID-19 turned to outbreak very fast. As of February 4, 2020, the new growth speed of diagnosis rates had reached $20 \%$ in China, especially in Hubei Province, which was more serious than any other areas, and the number of growth speed reached 35\% (all the data were collected from the official website of National Health Commission of the People's Republic of China). Diversities among different periods of COVID-19 had been reported [11]. Furthermore, a newly published meta-analysis showed that, lymphopenia, decreased albumin, increased level of C-reactive protein (CRP) and Erythrocyte sedimentation rate (ESR), as well as Lactate dehydrogenase (LDH), seemed to be the most common abnormal laboratory findings [8]. The clinical features of the disease in outbreak phage were still not clear, so we did a descriptive research to state the clinical characteristics and inflammatory indexes of the outbreaking COVID-19.

In this study, we aimed to describe the disease characteristics and figure out the relationship between the disease severity, clinical characteristic and inflammatory cytokines.

\section{Materials and Methods}

\section{Patients}

Our institutional review board approved this retrospective study (KYLL-2020-104). From February 3, 2020, to March 20, 2020, we conducted a retrospective study focusing on the clinical characteristics of confirmed cases of COVID-19 in Tongji Hospital, Tongji Medical College, Huazhong University of Science and Technology, during which the rapid growth speed of diagnosis rated up to $58 \%$ in Wuhan, Hubei province. Case definitions of confirmed human infection were in accordance with the COVID-19 Guidelines (trial version 7) from the National Health Committee of the People's Republic of China. COVID-19 patients were diagnosed through the symptoms and computed tomography (CT) image combining the detection of 2019-nCoV RNA or the specific virus IgM and IgG antibody to COVID-19.

We collected data of 88 patients admitted to our hospital with confirmed COVID-19 in Wuhan, Hubei province and extracted the medical records of patients in Tongji Hospital. The data included demographic data, exposure history, medical histories, symptoms, signs, laboratory findings and chest CT image. The severity classifying criteria was based on a previous study [12]. In brief, the patients were classified by typical symptoms, radiology manifestation, respiratory rates, pulse oxygen saturation, oxygenation index and existing of respiratory or circulatory failure. A team of doctors who had been treating patients with COVID-19 collected and reviewed the data. Because of the urgent need to collect data on this emerging novel pathogen, the written informed consent was waived. We used a standardized case report form to retrospectively collect the clinical and laboratory data.

\section{Laboratory tests}

All the respiratory and blood specimens were collected at admission. Nasopharyngeal swab specimens collected from all patients were tested by real time polymerase chain reaction (RT-PCR) for 2019-nCoV RNA. And the serum was collected to detect the specific IgM and IgG antibody to COVID-19. Other laboratory tests included complete blood cell count, hepatorenal function (alanine aminotransferase [ALT], aspartate aminotransferase [AST], globulin, LDH, creatinine and urea nitrogen), electrolytes, coagulation profile, myocardial enzyme and inflammatory cytokines (CRP, ferritin, Interleukin [IL]-1 $\beta$, IL-2R, IL-6, IL-8, IL-10, Tumor Necrosis Factor [TNF]- $\alpha$ ). All the detection was finished in the key laboratory of Tongji Hospital.

\section{Statistical analysis}

We summarized continuous variables as either the mean, standard deviation (SD) or median with interquartile range (IQR). For categorical variables, we calculated the percentages of patients in each category. Comparisons between different groups were made using Student $t$ test or Mann-Whitney $U$ test for continuous data, and the $\chi^{2}$ or Fisher exact test for categorical data. Correlation coefficients were then calculated between clinical and laboratory findings using Spearman or Pearson correlation as appropriate. All statistical analysis procedures were 
conducted using SPSS 22.0 software (IBM, Armonk, $\mathrm{NY}), p<0.05$ was considered statistically significant.

\section{Results}

\section{Clinical characteristics and laboratory parameters}

The study population included 88 confirmed and hospitalized COVID-19 patients. The results showed that average age was 57.11 years (SD, \pm 15.39 ), and $36(40.91 \%)$ were men. $32(36.36 \%)$ patients were old people ( $\geq 65$ years). The median body mass index (BMI) was 24.03 (IQR, 21.64-26.61; range 15.05-32.39). $10(11.36 \%)$ patients had definite exposure history and 3 of these patients were clustered. Of these 88 patients, $68(77.27 \%)$ of them were tested with positive viral nucleic acid test and $20(22.73 \%)$ of them with specific virus IgM and IgG antibody to COVID-19. The median duration from disease onset to hospital admissions were 11 days (IQR, 6.50-14.50). Among these patients, $41(46.59 \%)$ had 1 or more existing comorbidity, including hypertension (23 [26.14\%]), diabetes mellitus (DM) (11 [12.50\%]), coronary atherosclerotic heart disease (CAD) (7 [7.95\%]), chronic obstructive pulmonary disease (COPD) (4 $[4.55 \%])$, and malignancy $(4[4.55 \%])$. The most common manifestations of COVID-19, at the onset of the disease, were fever (63 [71.59\%]), cough (52 [59.09\%]), dyspnea (34 [38.64\%]), fatigue (26 [29.55\%]) and diarrhea (22 [25.00\%]). Additional symptoms included myalgia, chill, headache, expectoration, vomiting, haemoptysis and pharyngalgia (Table 1).

The blood counts of 8 of the $88(9.09 \%)$ patients showed leucopenia (white blood cell count (WBC) $\left.<4 \times 10^{9} / \mathrm{L}\right), \quad 44(50.00 \%)$ showed lymphocytopenia (lymphocyte count $\left.<1.10 \times 10^{9} / \mathrm{L}\right), 44(50.00 \%)$ showed eosinoponia (eosinophil count $<0.02 \times 10^{9} / \mathrm{L}$ ) and 16 $(18.18 \%)$ showed mononucleosis (monocyte count $>0.60 \times 10^{9} / \mathrm{L}$ ). As for inflammatory cytokines, the levels of serum CRP and ferritin increased in 59 $(67.05 \%)$ and $43(48.86 \%)$ patients, $5(5.68 \%)$ patients had higher serum levels of IL-1 $\beta(>5.0 \mathrm{pg} / \mathrm{mL}) .9$ $(10.23 \%)$ patients had increased serum levels of IL-2R $(>710 \mathrm{U} / \mathrm{ml})$. There are $15(17.05 \%), 6(6.82 \%)$ and 4 $(4.55 \%)$ patients with higher levels of serum IL-6 (>7.0 $\mathrm{pg} / \mathrm{ml})$, IL-8 (>62 pg/ml) and IL-10 $(>9.1 \mathrm{pg} / \mathrm{ml})$. Besides, levels of serum TNF-a increased in 11 (12.50\%) patients. For liver function parameters, levels of ALT and AST increased in 29 (32.95\%) and 32 $(36.36 \%)$ patients, levels of globulin and LDH increased in $19(21.59 \%)$ and $48(54.55 \%)$ patients. The levels of D-dimer and prothrombin time (PT) increased in $40(45.45 \%)$ and $26(29.55 \%)$ patients. The results indicated that there were $29(36.36 \%)$ patients occurring liver damage, 7 (7.95\%) patients occurring myocardial damage which was diagnosed by serum cardiac troponin I (cTnI) elevation $(>15.6 \mu \mathrm{g} / \mathrm{L})$, and 2 patients $(2.27 \%)$ occurring acute kidney injury in our study with the serum creatinine above $104 \mu \mathrm{mol} / \mathrm{L}$ (Table 2).

Table 1. Demographics and baseline characteristics of patients infected with 2019-nCoV

\begin{tabular}{|c|c|}
\hline Clinical Characteristics & Patients $(n=88)$ \\
\hline \multicolumn{2}{|l|}{ Age } \\
\hline Mean age (SD) & $57.11(15.39)$ \\
\hline Range & $21-89$ \\
\hline \multicolumn{2}{|l|}{ Distribution no. (\%) } \\
\hline$<65$ & $56(63.64 \%)$ \\
\hline$\geq 65$ & $32(36.36 \%)$ \\
\hline \multicolumn{2}{|l|}{ Gender no. (\%) } \\
\hline Male & $36(40.91 \%)$ \\
\hline Female & $52(59.09 \%)$ \\
\hline \multicolumn{2}{|l|}{ BMI } \\
\hline Median (IQR) & $24.12(21.72-25.94)$ \\
\hline \multicolumn{2}{|l|}{ Distribution no. (\%) } \\
\hline$<18.5$ & $3(3.41 \%)$ \\
\hline $18.5-24$ & $34(38.64 \%)$ \\
\hline$\geq 24$ & $43(48.86 \%)$ \\
\hline \multicolumn{2}{|c|}{ Onset of symptom to Hospital admission } \\
\hline Median days (IQR) & $11.00(6.50-14.50)$ \\
\hline Any comorbidity (\%) & $41(46.59 \%)$ \\
\hline Hypertension & $23(26.14 \%)$ \\
\hline Diabetes mellitus & $11(12.50 \%)$ \\
\hline CAD & $7(7.95 \%)$ \\
\hline COPD & $4(4.55 \%)$ \\
\hline Malignancy & $4(4.55 \%)$ \\
\hline Hyperthyroidism & $2(2.27 \%)$ \\
\hline Depression & $1(1.14 \%)$ \\
\hline Gallstones & $1(1.14 \%)$ \\
\hline Gout & $1(1.14 \%)$ \\
\hline Parkinson disease & $1(1.14 \%)$ \\
\hline Cerebral infarction & $1(1.14 \%)$ \\
\hline Kidney stones & $1(1.14 \%)$ \\
\hline \multicolumn{2}{|l|}{ Signs and symptoms (\%) } \\
\hline Fever & $63(71.59 \%)$ \\
\hline Cough & $52(59.09 \%)$ \\
\hline Dyspnea & $34(38.64 \%)$ \\
\hline Fatigue & $26(29.55 \%)$ \\
\hline Diarrhea & $22(25.00 \%)$ \\
\hline Myalgia & $21(23.86 \%)$ \\
\hline Expectoration & $15(17.05 \%)$ \\
\hline Chill & $12(13.64 \%)$ \\
\hline Headache & $12(13.64 \%)$ \\
\hline Pharyngalgia & $5(5.68 \%)$ \\
\hline Nausea and vomiting & $4(4.55 \%)$ \\
\hline Haemoptysis & $2(2.27 \%)$ \\
\hline \multicolumn{2}{|c|}{ Duration of 2019-nCoV nucleic acid } \\
\hline Median no. days (IQR) & $22.00(16.00-27.25)$ \\
\hline Outcome & $86(97.73 \%)$ \\
\hline Acute exacerbation & $10(11.36 \%)$ \\
\hline Discharged & $76(86.36 \%)$ \\
\hline
\end{tabular}

\section{The relationship between clinical characteristics and disease severity}

As shown in Table 3, we divided 88 hospitalized patients into moderate (47 [53.41\%]), severe (32 [36.36\%]) and critically ill (9 [10.23\%]) groups according to the classifying criteria. Interestingly, men 
were the majority (7 [77.78\%]) of the critically ill group patients, while women $(52$ [59.09\%]) seemed slightly more in overall patients. It deserved to be mentioned that the average age was ascending along with the increasing disease severity (52.49 years vs 59.94 years vs 74.78 years, $p<0.001$ ). BMI seemed to decrease along with the increasing disease severity, the median BMI of moderate, severe and critically ill group were 24.22 (IQR, 21.75-25.89), 24.03 (IQR, 22.04-27.35) and 21.88 (IQR, 16.24-25.45), while it is non-Significant statistically $(p=0.340)$. A total of 41 $(46.59 \%)$ patients existed comorbidities. There were 17 $(36.17 \%)$ patients with comorbidities in the moderate group $(n=47)$, in the severe group $(n=32)$ the number is $17(53.13 \%)$, and there were $7(77.78 \%)$ patients with comorbidities in critically ill group $(n=9)$, which demonstrated the increasing rates of comorbidities in severe and critically ill patients, and there was a dominant $p$ value $(p=0.047)$ about comorbidity occurrence among these three groups. Concerning the clinical symptoms, we found there was no dominating diversity between different severities of COVID-19.

\section{The relationship between laboratory parameters and disease severity}

To determine the major differences of clinical features among different group patients, we explored the difference of laboratory parameters according to disease severity (Table 4).

Table 2. Laboratory findings of patients infected with 2019-nCoV on admission to hospital

\begin{tabular}{|c|c|}
\hline Laboratory findings & Patients $(\mathrm{n}=88)$ \\
\hline \multicolumn{2}{|c|}{ WBC count $(3.5-9.5 \times 109 / \mathrm{L})$} \\
\hline Median (IQR) & $5.41(3.86-6.77)$ \\
\hline \multicolumn{2}{|l|}{ Distribution no. (\%) } \\
\hline$<3.50$ & $8(9.09 \%)$ \\
\hline $3.50-9.50$ & $52(59.09 \%)$ \\
\hline$>9.50$ & $5(5.68 \%)$ \\
\hline \multicolumn{2}{|c|}{ Lymphocyte count $(1.1-3.2 \times 109 / \mathrm{L})$} \\
\hline Median (IQR) & $1.08(0.71-1.50)$ \\
\hline \multicolumn{2}{|l|}{ Distribution no. (\%) } \\
\hline$<1.10$ & $44(50.0 \%)$ \\
\hline $1.10-3.20$ & $42(47.73 \%)$ \\
\hline \multicolumn{2}{|c|}{ Eosinophil count $(0.02-0.52 \times 109 / \mathrm{L})$} \\
\hline Median (IQR) & $0.01(0.00-0.10)$ \\
\hline \multicolumn{2}{|l|}{ Distribution no. (\%) } \\
\hline$<0.02$ & $44(50.0 \%)$ \\
\hline $0.02-0.52$ & $40(45.45 \%)$ \\
\hline$>0.52$ & $1(1.14 \%)$ \\
\hline \multicolumn{2}{|c|}{ Monocyte count $(0.10-0.60 \times 109 / \mathrm{L})$} \\
\hline Median (IQR) & $0.40(0.32-0.56)$ \\
\hline \multicolumn{2}{|l|}{ Distribution no. (\%) } \\
\hline $0.10-0.60$ & $70(79.55 \%)$ \\
\hline$>0.60$ & $16(18.18 \%)$ \\
\hline \multicolumn{2}{|l|}{ CRP (<1 mg/L) } \\
\hline Median (IQR) & $14.70(2.38-67.05)$ \\
\hline \multicolumn{2}{|l|}{ Distribution no. (\%) } \\
\hline$<1.00$ & $6(6.82 \%)$ \\
\hline$\geq 1.00$ & $59(67.05 \%)$ \\
\hline
\end{tabular}

\begin{tabular}{|c|c|}
\hline Laboratory findings & Patients $(n=88)$ \\
\hline Median (IQR) & $24.00(15.00-47.00)$ \\
\hline \multicolumn{2}{|l|}{ Distribution no. (\%) } \\
\hline$\leq 33$ & $56(63.64 \%)$ \\
\hline$>33$ & $29(32.95 \%)$ \\
\hline \multicolumn{2}{|l|}{ AST $(\leq 32 \mathrm{U} / \mathrm{L})$} \\
\hline Median (IQR) & $27.00(20.00-44.00)$ \\
\hline \multicolumn{2}{|l|}{ Distribution no. (\%) } \\
\hline$\leq 32$ & $33(37.50 \%)$ \\
\hline$\geq 32$ & $32(36.36 \%)$ \\
\hline \multicolumn{2}{|l|}{ Globulin (20-35 g/L) } \\
\hline Median (IQR) & $31.30(29.35-34.65)$ \\
\hline \multicolumn{2}{|l|}{ Distribution no. (\%) } \\
\hline $20-35$ & $66(75.00 \%)$ \\
\hline$>35$ & $19(21.59 \%)$ \\
\hline \multicolumn{2}{|l|}{ LDH (135-214 U/L) } \\
\hline Median (IQR) & $251.0(194.5-333.0)$ \\
\hline \multicolumn{2}{|l|}{ Distribution no. (\%) } \\
\hline$<214$ & $37(42.05 \%)$ \\
\hline$\geq 214$ & $48(54.55 \%)$ \\
\hline \multicolumn{2}{|c|}{ Ferritin (15-150 ng/ml) } \\
\hline Median (IQR) & $412.6(158.4-952.2)$ \\
\hline \multicolumn{2}{|l|}{ Distribution no. (\%) } \\
\hline$<150$ & $14(15.91 \%)$ \\
\hline$\geq 150$ & $43(48.86 \%)$ \\
\hline \multicolumn{2}{|l|}{$\operatorname{IL}-1 \beta(<5.0 \mathrm{pg} / \mathrm{ml})$} \\
\hline \multicolumn{2}{|l|}{ Distribution no. (\%) } \\
\hline$<5.0$ & $47(53.41 \%)$ \\
\hline$\geq 5.0$ & $5(5.68 \%)$ \\
\hline \multicolumn{2}{|l|}{ IL-2R (223-710 U/ml) } \\
\hline Median (IQR) & $437.5(253.5-578.8)$ \\
\hline \multicolumn{2}{|l|}{ Distribution no. (\%) } \\
\hline$<223$ & $11(12.50 \%)$ \\
\hline $223-710$ & $34(38.64 \%)$ \\
\hline$>710$ & $9(10.23 \%)$ \\
\hline \multicolumn{2}{|l|}{$\operatorname{IL}-6(<7.0 \mathrm{pg} / \mathrm{ml})$} \\
\hline Median (IQR) & $3.02(1.50-11.47)$ \\
\hline Distribution no. (\%) & \\
\hline$<7$ & $38(43.18 \%)$ \\
\hline$\geq 7$ & $15(17.05 \%)$ \\
\hline IL-8 $(<62$ pg/ml) & \\
\hline Median (IQR) & $8.75(5.00-13.48)$ \\
\hline Distribution no. (\%) & \\
\hline$<62$ & 48 (54.55\%) \\
\hline$\geq 62$ & $6(6.82 \%)$ \\
\hline IL-10 (< 9.1 pg/ml) & \\
\hline Distribution no. (\%) & \\
\hline$<9.1$ & 47 (53.41\%) \\
\hline$\geq 9.1$ & $4(4.55 \%)$ \\
\hline TNF-a $(<8.1 \mathrm{pg} / \mathrm{ml})$ & \\
\hline Median (IQR) & $7.20(5.70-7.20)$ \\
\hline Distribution no. (\%) & \\
\hline$<8.1$ & $25(28.41 \%)$ \\
\hline$\geq 8.1$ & $11(12.50 \%)$ \\
\hline D-dimer $(<0.50 \mu \mathrm{g} / \mathrm{n}$ & \\
\hline Median (IQR) & $0.61(0.37-1.20)$ \\
\hline Distribution no. (\%) & \\
\hline$<0.50$ & $39(44.32 \%)$ \\
\hline$\geq 0.50$ & $40(45.45 \%)$ \\
\hline PT (11.5-14.5 s) & \\
\hline Median (IQR) & $13.90(13.30-14.80)$ \\
\hline Distribution no. (\%) & \\
\hline$<14.5$ & $52(59.09 \%)$ \\
\hline$\geq 14.5$ & $26(29.55 \%)$ \\
\hline $\operatorname{cTnI}(<15.6 \mu \mathrm{g} / \mathrm{L})$ & \\
\hline Median (IQR) & $5.70(2.80-11.50)$ \\
\hline Distribution no. (\%) & \\
\hline$<15.6$ & $52(59.09 \%)$ \\
\hline$\geq 15.6$ & $7(7.95 \%)$ \\
\hline
\end{tabular}


Table 3. Demographics and baseline characteristics of patients infected with 2019-nCoV according to disease severity

\begin{tabular}{|c|c|c|c|c|}
\hline \multirow[t]{2}{*}{ Characteristics } & \multicolumn{3}{|l|}{ Disease Severity } & \multirow[t]{2}{*}{$p$ value } \\
\hline & Moderate $(\mathrm{n}=47)$ & Severe $(n=32)$ & Critically ill $(n=9)$ & \\
\hline \multicolumn{5}{|l|}{ Age } \\
\hline Mean age (SD) & $52.49(14.62)$ & $59.94(13.96)$ & $74.78(10.06)$ & $<0.001$ \\
\hline Distribution no. (\%) & & & & $<0.001$ \\
\hline$<65$ & $36 / 47(76.60)$ & $19 / 32(59.38)$ & $1 / 9(11.11)$ & - \\
\hline$\geq 65$ & $11 / 47(23.40)$ & $13 / 32(40.62)$ & $8 / 9(88.89)$ & - \\
\hline Gender no. (\%) & & & & 0.013 \\
\hline Male & $21 / 47(44.68)$ & $8 / 32(25.00)$ & $7 / 9(77.78)$ & - \\
\hline Female & $26 / 47(55.32)$ & $24 / 32(75.00)$ & $2 / 9(22.22)$ & - \\
\hline \multicolumn{5}{|l|}{ BMI } \\
\hline Median (IQR) & $24.22(21.75-25.89)$ & $24.03(22.04-27.35)$ & $21.88(16.24-25.45)$ & 0.340 \\
\hline \multicolumn{5}{|c|}{ Onset of symptom to Hospital admission } \\
\hline Median no. days (IQR) & $11.00(6.00-23.00)$ & $11.00(8.00-14.00)$ & $13.00(12.00-14.00)$ & 0.710 \\
\hline Any comorbidity (\%) & $17 / 47(36.17)$ & $17 / 32(53.13)$ & $7 / 9(77.78)$ & 0.047 \\
\hline Hypertension & $10(58.82)$ & $10(58.82)$ & $3(42.86)$ & 0.535 \\
\hline Diabetes mellitus & $6(35.29)$ & $4(23.53)$ & $1(14.29)$ & 0.991 \\
\hline $\mathrm{CAD}$ & $3(17.65)$ & $3(17.65)$ & $1(14.29)$ & 0.831 \\
\hline COPD & $1(5.88)$ & $2(11.76)$ & $1(14.29)$ & 0.419 \\
\hline Malignancy & $2(11.76)$ & $1(5.88)$ & $1(14.29)$ & 0.591 \\
\hline \multicolumn{5}{|l|}{ Signs and symptoms (\%) } \\
\hline Fever & $32(68.09)$ & $24(75.00)$ & $7(77.78)$ & 0.728 \\
\hline Cough & $31(65.96)$ & $18(56.25)$ & $3(33.33)$ & 0.174 \\
\hline Dyspnea & $13(27.66)$ & $16(50.00)$ & $5(55.56)$ & 0.074 \\
\hline Fatigue & $15(31.91)$ & $10(31.25)$ & $1(11.11)$ & 0.440 \\
\hline Diarrhea & $7(14.89)$ & $13(40.63)$ & $2(22.22)$ & 0.034 \\
\hline \multicolumn{5}{|c|}{ Duration of 2019- nCoV nucleic acid } \\
\hline Median no. days (IQR) & $21.00(15.50-24.50)$ & $23.00(18.75-28.00)$ & $13.00(5.00-29.00)$ & 0.129 \\
\hline Outcome & $47(100.00)$ & $31(96.88)$ & $8(88.89)$ & $<0.001$ \\
\hline Discharged & $46(97.87)$ & $30(93.75)$ & $0(0)$ & \\
\hline Acute exacerbation & $1(2.13)$ & $1(3.13)$ & $8(88.89)$ & \\
\hline
\end{tabular}

CAD, Coronary atherosclerotic heart disease. COPD, Chronic obstructive pulmonary disease.

The results showed that lymphocyte count $(1.34$ vs 0.89 vs $0.57, p<0.001)$, eosinophil count $(0.07$ vs 0.00 vs $0.00, p=0.002)$, CRP (7.20 vs 33.20 vs $98.60, p<0.001)$, LDH (201.5 vs 297.0 vs 493.0, $\mathrm{p}<0.001)$ and PT (13.65 vs 14.20 vs $15.80, p=0.019$ ) were statistically significant among the three groups. Moreover, inflammatory cytokines obviously changed among these patients, including ferritin (348.5 vs 504.0 vs $1853.0, p=0.002)$, IL-2R (297.0 vs 478.0 vs 968.0, $p=0.005)$, IL-6 (1.88 vs 2.96 vs $34.01, p=0.002)$, IL-8 (7.10 vs 9.25 vs 76.10 , $p=0.003)$ and TNF-a (6.80 vs 6.60 vs $11.50 p=0.008)$. Patients in critically ill group were more likely to have lymphocytopenia $(85.71 \%$ vs $68.75 \%$ vs $34.04 \%$, $p=0.002)$ and eosinoponia $(31.91 \%$ vs $75.00 \%$ vs $83.33 \%, p=0.002)$, and were more likely to have higher IL-2R $(71.43 \%$ vs $11.11 \%$ vs $6.90 \%, p<0.001)$, IL-6 ( $83.33 \%$ vs $27.78 \%$ vs $17.24 \%, p=0.005)$, IL-8 ( $57.14 \%$ vs $5.56 \%$ vs $3.45 \%, p<0.001)$, IL-10 (33.33\% vs $5.56 \%$ vs $3.70 \%, p=0.046)$, TNF-a $(71.43 \%$ vs $35.29 \%$ vs 0 , $p=0.008)$, LDH ( $100 \%$ vs $75.00 \%$ vs $36.96 \%, p<0.001)$ and cTnI $(42.86 \%$ vs $9.52 \%$ vs $6.45 \%, p=0.025)$. The WBC count, monocyte count, globulin, and ALT showed no difference among these three groups. Though the changing levels among three groups seemed non-significant statistically, we still noticed that some parameters increasing apparently in the severe or critically ill group patients especially, such as IL-10 $(p=0.046)$ and D-dimer $(p=0.028)$, of which the median value and IQR were non-significant but the distribution is disparate.

\section{The relationship between clinical characteristics and inflammatory cytokines}

Then our group made correlation analysis to illuminate the relations between inflammatory indexes and other clinical parameters. Among all the inflammatory indexes, CRP $(r=0.262, p=0.015)$, LDH $(r=0.273, p=0.011)$, ferritin $(r=0.335, p=0.011), \quad$ IL-2R $(r=0.460, p=0.005), \quad$ IL-6 $\quad(r=0.300, p=0.029), \quad$ IL-8 $(r=0.325, p=0.017), \mathrm{IL}-10(r=0.342, p=0.025)$ and TNF- $\mathrm{a}$ $(r=0.429, p=0.001)$ were positively correlated with age (Figure 1). The result also indicated that BMI showed positive correlation with IL-2R $(r=-0.322, p=0.022)$, IL-6 $(r=-0.337, p=0.017)$, IL-10 $(r=-0.402, p=0.005)$ and TNF- $\alpha(r=-0.331, p=0.019)$ (Figure 2). Furthermore, to comprehend whether there were relations between different symptoms and inflammatory indexes, our group compared each symptom to the indexes. Then we found decreasing lymphocyte count (0.84 vs 1.21 , $p=0.008)$, and increasing CRP ( 34.50 vs $10.90, p=0.036$ ), IL-6 (6.67 vs 2.04, $p=0.015)$, TNF-a $(7.90$ vs 5.80, $p=0.036)$, PT $(14.50$ vs $13.70, p<0.001)$ occurred frequently in the patients with dyspnea. And the patients with fever showed decreasing lymphocyte count $(0.93$ vs $1.33, p=0.049)$, eosinophil count $(0.01$ vs $0.07, p=0.021)$, and increasing CRP (28.85 vs 5.40, $p=0.031)$ and IL-8 (9.90 vs 7.10, $p=0.045)$ (Table 5). 
Table 4. Laboratory findings of patients infected with 2019-nCoV on admission to hospital according to disease severity

\begin{tabular}{|c|c|c|c|c|}
\hline \multirow[t]{2}{*}{ Laboratory findings } & \multicolumn{3}{|l|}{ Disease Severity } & \multirow[t]{2}{*}{$p$ value } \\
\hline & Moderate $(\mathrm{n}=47)$ & Severe $(n=32)$ & Critically ill $(n=9)$ & \\
\hline \multicolumn{5}{|c|}{ WBC count $\left(3.5-9.5 \times 10^{9} / \mathrm{L}\right)$} \\
\hline Median (IQR) & $5.06(3.87-6.49)$ & $5.24(3.74-6.80)$ & $6.15(5.40-8.91)$ & 0.174 \\
\hline Distribution no. $(\%)$ & $27(57.45)$ & $31(96.88)$ & $7(77.78)$ & 0.489 \\
\hline$<3.50$ & $4(14.81)$ & $4(12.90)$ & 0 & - \\
\hline $3.50-9.50$ & $19(70.37)$ & $26(83.87)$ & $7(100.00)$ & - \\
\hline$>9.50$ & $4(14.81)$ & $1(3.23)$ & 0 & - \\
\hline \multicolumn{5}{|c|}{ Lymphocyte count $\left(1.1-3.2 \times 10^{9} / \mathrm{L}\right)$} \\
\hline Median (IQR) & $1.34(0.85-1.83)$ & $0.89(0.64-1.24)$ & $0.57(0.50-0.93)$ & $<0.001$ \\
\hline Distribution no. (\%) & $47(100.00)$ & $32(100.00)$ & $7(77.78)$ & 0.002 \\
\hline$<1.10$ & $16(34.04)$ & $22(68.75)$ & $6(85.71)$ & - \\
\hline $1.10-3.20$ & $31(65.96)$ & $10(31.25)$ & $1(14.29)$ & - \\
\hline \multicolumn{5}{|c|}{ Eosinophil count $\left(0.02-0.52 \times 10^{9} / \mathrm{L}\right)$} \\
\hline Median (IQR) & $0.07(0.01-0.15)$ & $0.00(0.00-0.02)$ & $0.00(0.00-0.02)$ & $<0.001$ \\
\hline Distribution no. (\%) & $47(100.00)$ & $32(100.00)$ & $6(66.67)$ & 0.002 \\
\hline$<0.02$ & $15(31.91)$ & $24(75.00)$ & $5(83.33)$ & - \\
\hline $0.02-0.52$ & $31(65.96)$ & $8(25.00)$ & $1(16.67)$ & - \\
\hline$>0.52$ & $1(2.13)$ & 0 & 0 & \\
\hline \multicolumn{5}{|c|}{ Monocyte count $\left(0.10-0.60 \times 10^{9} / \mathrm{L}\right)$} \\
\hline Median (IQR) & $0.41(0.32-0.60)$ & $0.39(0.30-0.45)$ & $0.36(0.30-0.64)$ & 0.508 \\
\hline Distribution no. (\%) & $47(100.00)$ & $32(100.00)$ & $7(77.78)$ & 0.226 \\
\hline $0.10-0.60$ & $36(76.60)$ & $29(90.63)$ & $5(71.43)$ & - \\
\hline$>0.60$ & $11(23.40)$ & $3(9.38)$ & $2(28.57)$ & - \\
\hline \multicolumn{5}{|l|}{ CRP (<1 mg/L) } \\
\hline Median (IQR) & $7.20(1.60-31.30)$ & $33.20(3.63-102.40)$ & $98.60(61.20-142.20)$ & $<0.001$ \\
\hline Distribution no. (\%) & $27(57.45)$ & $31(96.88)$ & $7(77.78)$ & 0.347 \\
\hline$<1.00$ & $3(11.11)$ & $3(9.68)$ & 0 & - \\
\hline$\geq 1.00$ & $24(88.89)$ & $28(90.32)$ & $7(100.00)$ & - \\
\hline \multicolumn{5}{|l|}{$\operatorname{ALT}(\leq 33 \mathrm{U} / \mathrm{L})$} \\
\hline Median (IQR) & $22.50(14.00-46.50)$ & $24.00(16.00-38.00)$ & $30.00(16.00-54.00)$ & 0.524 \\
\hline Distribution no. (\%) & $46(97.87)$ & $32(100.00)$ & $7(77.78)$ & 0.633 \\
\hline$\leq 33$ & $29(63.04$ & $23(71.88)$ & $4(57.14)$ & - \\
\hline$>33$ & $17(36.96)$ & $9(28.12)$ & $3(42.86)$ & - \\
\hline \multicolumn{5}{|l|}{ AST $(\leq 32$ U/L) } \\
\hline Median (IQR) & $23.50(17.75-34.25)$ & $28.00(21.00-54.50)$ & $44.00(41.00-56.00)$ & 0.002 \\
\hline Distribution no. (\%) & $27(57.45)$ & $31(96.88)$ & $7(77.78)$ & 0.002 \\
\hline$\leq 32$ & $15(55.56)$ & $18(58.06)$ & 0 & - \\
\hline$>32$ & $12(44.44)$ & $13(41.94)$ & $7(100.00)$ & - \\
\hline \multicolumn{5}{|l|}{ Globulin $(20-35 \mathrm{~g} / \mathrm{L})$} \\
\hline Median (IQR) & $30.70(28.55-34.23)$ & $32.65(29.45-35.38)$ & $33.70(30.50-38.00)$ & 0.242 \\
\hline Distribution no. (\%) & $46(97.87)$ & $32(100.00)$ & $7(77.78)$ & 0.290 \\
\hline $20-35$ & $38(82.61)$ & $24(75.00)$ & $4(57.14)$ & - \\
\hline$>35$ & $8(17.39)$ & $8(25.00)$ & $3(42.86)$ & - \\
\hline LDH (135-214 U/L) & & & & \\
\hline Median (IQR) & $201.5(176.0-277.8)$ & $297.0(211.8-428.3)$ & $493.0(471.0-570.0)$ & $<0.001$ \\
\hline Distribution no. (\%) & $46(97.87)$ & $32(100.00)$ & $7(77.78)$ & $<0.001$ \\
\hline $135-214$ & $29(63.04)$ & $8(25.00)$ & 0 & - \\
\hline$\geq 214$ & $17(36.96)$ & $24(75.00)$ & $7(100.00)$ & - \\
\hline Ferritin $(15-150 \mathrm{ng} / \mathrm{m}$ & & & & \\
\hline Median (IQR) & 348.5 (136.3-590.9) & $504.0(148.3-956.0)$ & 1853 (1222-3004) & 0.002 \\
\hline Distribution no. (\%) & $33(70.21)$ & $19(59.38)$ & $5(55.56)$ & 0.409 \\
\hline$<150$ & $9(27.27)$ & $5(26.32)$ & 0 & - \\
\hline$\geq 150$ & $24(72.73)$ & $14(73.68)$ & $5(100.00)$ & - \\
\hline $\mathrm{IL}-1 \beta(<5.0 \mathrm{pg} / \mathrm{ml})$ & & & & \\
\hline Distribution no. (\%) & $27(57.45)$ & $18(56.25)$ & $7(77.78)$ & 0.7458 \\
\hline$<5.0$ & $24(88.89)$ & $17(94.44)$ & $6(85.71)$ & - \\
\hline$\geq 5.0$ & $3(11.11)$ & $1(5.56)$ & $1(14.29)$ & - \\
\hline IL-2R (223-710 U/ml) & & & & \\
\hline Median (IQR) & $297.0(200.5-476.5)$ & $478.0(296.5-565.5)$ & $968.0(638.0-2279)$ & 0.005 \\
\hline Distribution no. (\%) & $29(61.70)$ & $18(56.25)$ & $7(77.78)$ & $<0.001$ \\
\hline$<223$ & $8(27.59)$ & $2(11.11)$ & $1(14.29)$ & - \\
\hline $223-710$ & $19(65.52)$ & $14(77.78)$ & $1(14.29)$ & - \\
\hline$>710$ & $2(6.89)$ & $2(11.11)$ & $5(71.42)$ & - \\
\hline IL-6 $(<7.0 \mathrm{pg} / \mathrm{ml})$ & & & & \\
\hline Median (IQR) & $1.88(1.50-5.76)$ & $2.96(1.89-14.07)$ & $34.01(8.71-158.10)$ & 0.002 \\
\hline Distribution no. (\%) & $29(61.70)$ & $18(56.25)$ & $6(66.67)$ & 0.005 \\
\hline$<7$ & $24(82.76)$ & $13(72.22)$ & $1(16.67)$ & - \\
\hline$\geq 7$ & $5(17.24)$ & $5(27.78)$ & $5(83.33)$ & - \\
\hline IL-8 $(<62$ pg/ml $)$ & & & & \\
\hline Median (IQR) & $7.10(5.00-10.60)$ & $9.25(7.25-12.73)$ & $76.10(18.80-436.00)$ & 0.003 \\
\hline Distribution no. (\%) & $29(61.70)$ & $18(56.25)$ & $7(77.78)$ & $<0.001$ \\
\hline
\end{tabular}




\begin{tabular}{|c|c|c|c|c|}
\hline \multirow[t]{2}{*}{ Laboratory findings } & \multicolumn{3}{|l|}{ Disease Severity } & \multirow[t]{2}{*}{$p$ value } \\
\hline & Moderate $(n=47)$ & Severe $(n=32)$ & Critically ill $(n=9)$ & \\
\hline$<62$ & $28(96.55)$ & $17(94.44)$ & $3(42.86)$ & - \\
\hline$\geq 62$ & $1(3.45)$ & $1(5.56)$ & $4(57.14)$ & - \\
\hline \multicolumn{5}{|l|}{ IL-10 (< 9.1 pg/ml) } \\
\hline Distribution no. (\%) & $27(57.45)$ & $18(56.25)$ & $6(66.67)$ & 0.046 \\
\hline$<9.1$ & $26(96.30)$ & $17(94.44)$ & $4(66.67)$ & - \\
\hline$\geq 9.1$ & $1(3.70)$ & $1(5.56)$ & $2(33.33)$ & - \\
\hline \multicolumn{5}{|l|}{ TNF-a $(<8.1 \mathrm{pg} / \mathrm{ml})$} \\
\hline Median (IQR) & $6.80(5.40-7.60)$ & $6.60(5.10-10.50)$ & $11.50(8.50-22.50)$ & 0.008 \\
\hline Distribution no. (\%) & $12(25.53)$ & $17(53.13)$ & $7(77.78)$ & 0.008 \\
\hline$<8.1$ & $12(100.00)$ & $11(64.71)$ & $2(28.57)$ & - \\
\hline$\geq 8.1$ & 0 & $6(35.29)$ & $5(71.43)$ & - \\
\hline \multicolumn{5}{|c|}{ D-dimer $(<0.50 \mu \mathrm{g} / \mathrm{ml})$} \\
\hline Median (IQR) & $0.49(0.32-0.81)$ & $0.70(0.40-1.57)$ & $0.83(0.65-6.64)$ & 0.086 \\
\hline Distribution no. (\%) & $42(89.36)$ & $30(93.75)$ & $7(77.78)$ & 0.028 \\
\hline$<0.50$ & $26(61.90)$ & $12(40.00)$ & $1(14.29)$ & - \\
\hline$\geq 0.50$ & $16(38.10)$ & $18(60.00)$ & $6(85.71)$ & - \\
\hline \multicolumn{5}{|l|}{ PT (11.5-14.5 s) } \\
\hline Median (IQR) & $13.65(13.18-14.53)$ & $14.20(13.60-15.20)$ & $15.80(13.20-16.20)$ & 0.019 \\
\hline Distribution no. (\%) & $42(89.36)$ & $29(90.63)$ & $7(77.78)$ & 0.062 \\
\hline$<14.5$ & $31(73.81)$ & $19(65.52)$ & $2(28.57)$ & - \\
\hline$\geq 14.5$ & $11(26.19)$ & $10(34.48)$ & $5(71.43)$ & - \\
\hline \multicolumn{5}{|l|}{ cTn I $(<15.6 \mu \mathrm{g} / \mathrm{L})$} \\
\hline Median (IQR) & $3.30(2.63-5.65)$ & $6.30(3.23-12.55)$ & $8.70(6.30-70.70)$ & 0.008 \\
\hline Distribution no. (\%) & $31(65.96)$ & $21(65.63)$ & $7(77.78)$ & 0.025 \\
\hline$<15.6$ & $29(93.55)$ & $19(90.48)$ & $4(57.14)$ & - \\
\hline$\geq 15.6$ & $2(6.45)$ & $2(9.52)$ & $3(42.86)$ & - \\
\hline
\end{tabular}

WBC, White blood cell. CRP, C-reactive protein. ALT, Alanine aminotransferase. AST, Aspartate aminotransferase. LDH, Lactate dehydrogenase. IL, Interleukin. TNF,

Tumor Necrosis Factor. PT, Prothrombin time. cTnI, cardiac troponin I.

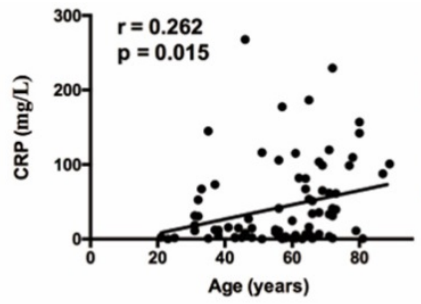

A

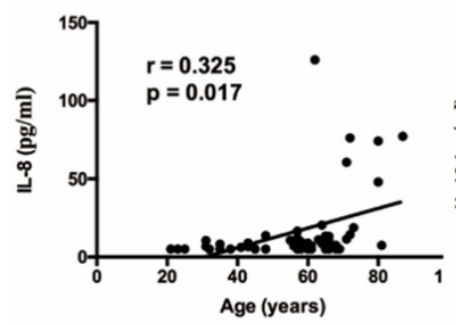

E

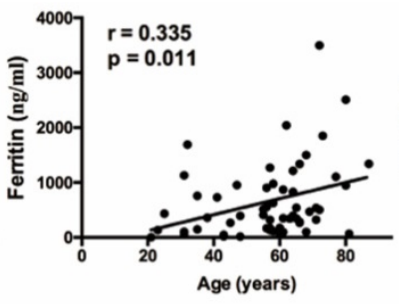

B

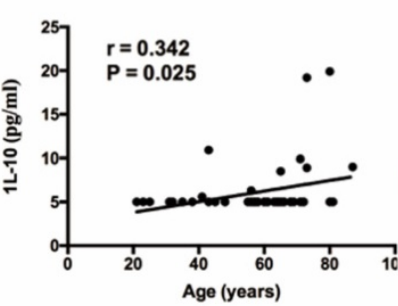

F

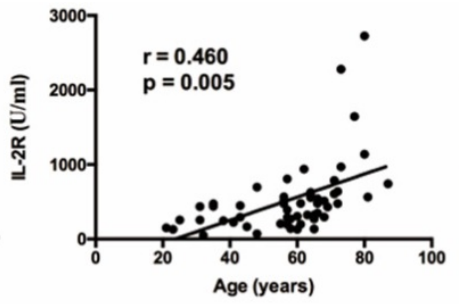

C

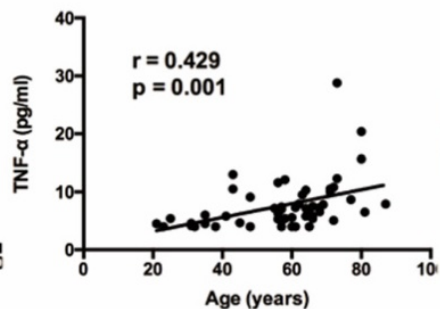

G

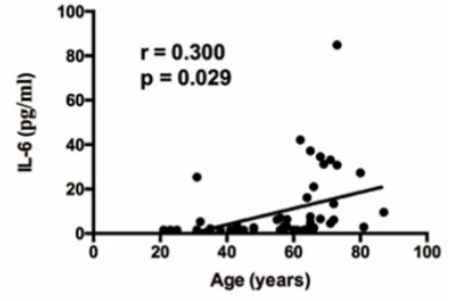

D

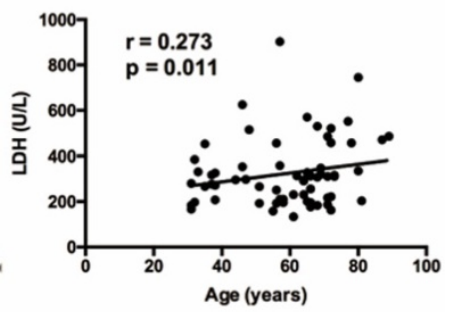

H

Figure 1. The relationship between age and inflammatory cytokines. (A) The relationship between age and CRP. (B) The relationship between age and ferritin. (C) The relationship between age and IL-2R. (D) The relationship between age and IL-6. (E) The relationship between age and IL-8. (F) The relationship between age and IL-10. (G) The relationship between age and TNF-a. (H) The relationship between age and LDH. CRP, C-reactive protein. IL, Interleukin. TNF, Tumor Necrosis Factor. LDH, Lactate dehydrogenase.

\section{Prognostic factors in COVID-19 patients}

Our data collection was up to March 20. All the 47 patients in the mild ill group were discharged. In the severe ill group, there was 1 patient turned into critical patient and admitted to intensive care unit with mechanical ventilation, 3 patients were still in the hospital for treatment and the other 28 patients left hospital. In the critically ill group, 2 patients left hospital, 2 patients were still alive with mechanical ventilation, and 4 patients died. The median duration of 2019-nCoV nucleic acid was 22.00 days (IQR, 16.00-27.25). The ICU- and death patients were usually deemed to be caused by acute exacerbation of COVID-19, and there were 10 patients in our study that suffered from COVID-19 exacerbation. To elucidate what influenced the progression of the disease exacerbation, we made a one-way analysis of variance and discovered that gender, age, disease severity, underlying diseases and several laboratory 
parameters (AST, LDH, IL-6, IL-2R, IL-8, D-dimer and CTnI) affected COVID-19 progress statically significantly (Table 6).

\section{Discussion}

Our study was a descriptive research of 88 COVID-19 hospitalized patients in Wuhan, China. As mentioned above, the study was designed to demonstrate the clinical features of COVID-19 in the outbreak phage comparing to the previous data in initiate phage, and found the latent connections between considerable baseline information or laboratory indicators and the severity of the disease.
Then we found several hints to the severity of COVID-19 so as to assist the clinicians to recognize severe patients timely and focus on the expectant treatment for each patient.

The epidemiologic characteristic was not definitely in our research, because the patients were collected in the outbreak stage of COVID-19 and the disease spread rapidly. Amounts of patients were infected in daily life and public environment, such as market or restaurant, which led to the simultaneously outbreak and unclearly exposure history.
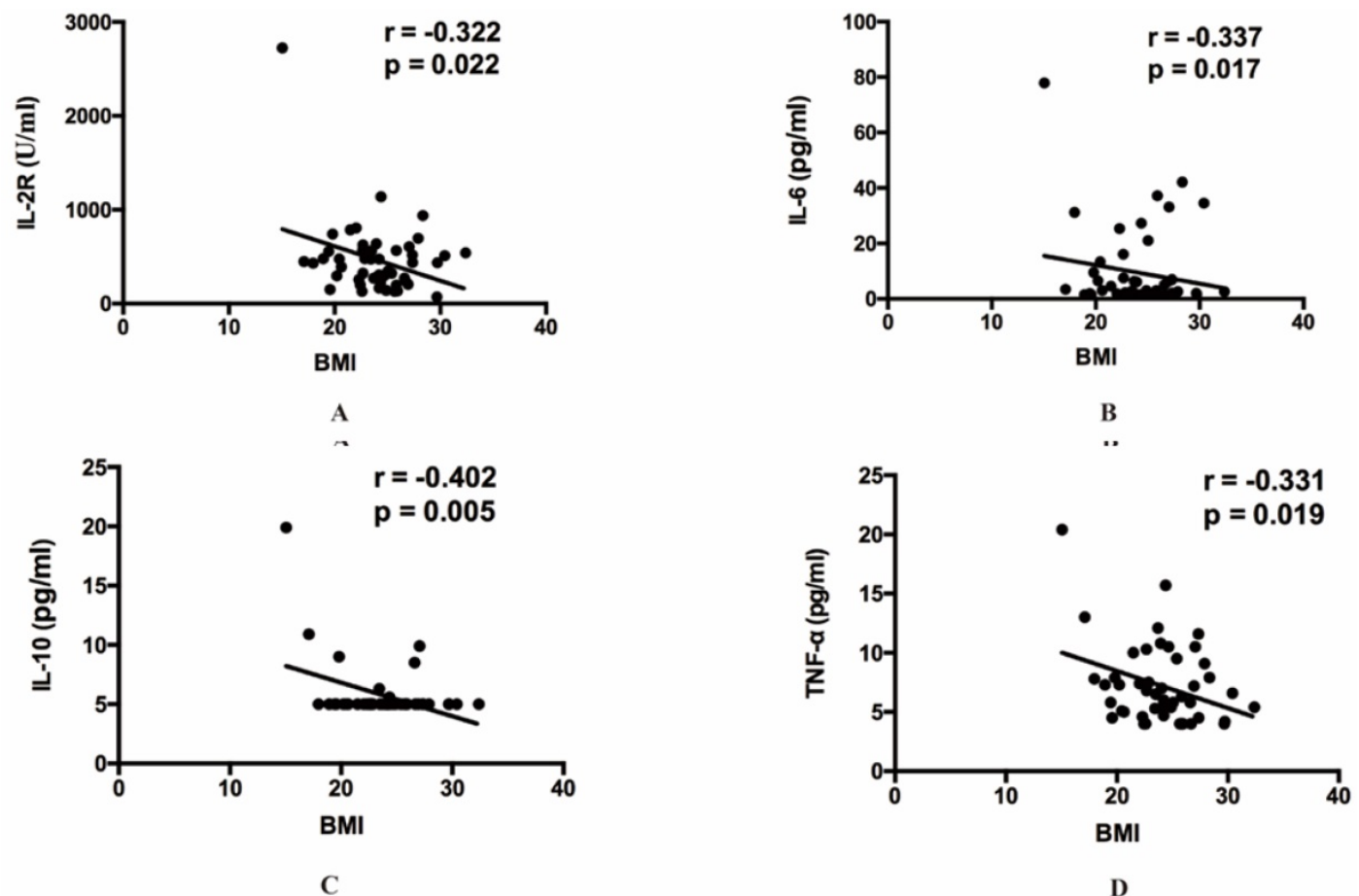

Figure 2. The relationship between BMI and inflammatory cytokines. (A) The relationship between BMI and IL-2R. (B) The relationship between BMI and IL-6. (C) The relationship between BMI and IL-10. (D) The relationship between BMI and TNF- $\alpha$. IL, Interleukin. TNF, Tumor Necrosis Factor.

Table 5. The relations between different symptoms and inflammatory indexes

\begin{tabular}{|c|c|c|c|c|c|c|}
\hline & \multicolumn{2}{|l|}{ Dyspnea } & \multirow[t]{2}{*}{$p$} & \multicolumn{2}{|l|}{ Fever } & \multirow[t]{2}{*}{$p$} \\
\hline & Yes $(n=34)$ & No $(n=54)$ & & Yes $(n=63)$ & No $(n=25)$ & \\
\hline \multicolumn{7}{|c|}{ Lymphocyte count $\left(1.1-3.2 \times 10^{9} / \mathrm{L}\right)$} \\
\hline Median (IQR) & $0.84(0.54-1.34)$ & $1.21(0.85-1.67)$ & 0.008 & $0.93(0.68-1.35)$ & $1.33(0.90-1.81)$ & 0.049 \\
\hline \multicolumn{7}{|c|}{ Eosinophil count $\left(0.02-0.52 \times 10^{9} / \mathrm{L}\right)$} \\
\hline Median (IQR) & $0.01(0.00-0.08)$ & $0.02(0.00-0.14)$ & 0.126 & $0.01(0.00-0.09)$ & $0.07(0.01-0.15)$ & 0.021 \\
\hline \multicolumn{7}{|l|}{$\mathrm{CRP}(<1 \mathrm{mg} / \mathrm{L})$} \\
\hline $\begin{array}{l}\text { Median (IQR) } \\
\text { IL-6< }<7.0 \mathrm{pg} / \mathrm{n}\end{array}$ & $34.50(5.00-90.35)$ & $10.90(1.70-48.65)$ & 0.036 & $28.85(3.68-81.28)$ & $5.40(1.10-34.65)$ & 0.031 \\
\hline $\begin{array}{l}\text { Median (IQR) } \\
\text { IL-8 }<62 \mathrm{pg} / \mathrm{m}\end{array}$ & $6.67(2.25-26.81)$ & $2.04(1.50-5.76)$ & 0.015 & $5.04(1.60-25.36)$ & $2.26(1.50-5.13)$ & 0.064 \\
\hline Median (IQR) & $9.60(7.25-54.20)$ & $7.10(5.00-12.70)$ & 0.109 & $9.90(5.38-20.00)$ & $7.10(5.00-9.63)$ & 0.045 \\
\hline \multicolumn{7}{|c|}{ TNF-a $(<8.1 \mathrm{pg} / \mathrm{ml})$} \\
\hline Median (IQR) & $7.90(5.50-11.20)$ & $5.80(4.60-7.35)$ & 0.036 & $6.90(4.63-10.25)$ & $6.05(5.25-7.50)$ & 0.436 \\
\hline \multicolumn{7}{|l|}{ PT (11.5-14.5 s) } \\
\hline
\end{tabular}


Table 6. Analysis of factors influencing the prognosis of patients infected with 2019-nCoV

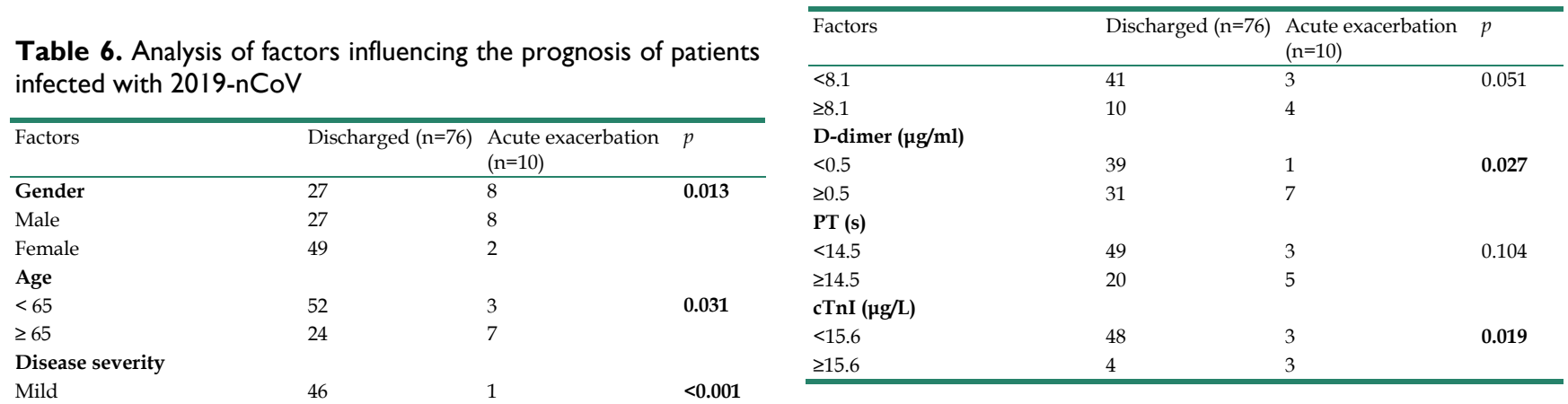

Mild

Critically ill

BMI

$<18.5$

$18.5-24$

$>24$

Any comorbidity

Yes

No

Glucocorticoid use

Yes

No

WBC count $\left(\times 10^{9} / \mathrm{L}\right)$

$<3.5$

3.5-9.5

$>9.5$

Lymphocyte count $\left(\times 10^{9} / \mathrm{L}\right)$

$<1.1$

1.1-3.2

Eosinophil count $\left(\times 10^{9} / \mathrm{L}\right)$

$<0.02$

$0.02-0.52$

$>0.52$

Monocyte count $\left(\times 10^{9} / \mathrm{L}\right)$

0.1-0.6

$>0.6$

CRP (mg/L)

$<1.0$

$\geq 1.0$

ALT (U/L)

$<33$

$\geq 33$

AST (U/L)

$<32$

$\geq 32$

Globulin $(\mathrm{g} / \mathrm{L})$

20-35

$>35$

LDH (U/L)

$<214$

$\geq 214$

Ferritin (ng/ml)

$<150$

$\geq 150$

IL-1 $\beta(\mathrm{pg} / \mathrm{ml})$

$<5.0$

$\geq 5.0$

IL-6 (pg/ml)

$<7.0$

$\geq 7.0$

IL-2R (U/ml)

$<223$

223-710

$>710$

IL-8 (pg/ml)

$<62$

$\geq 62$

IL-10 (pg/ml)

$<9.1$

$\geq 9.1$

TNF- $\alpha(\mathrm{pg} / \mathrm{ml})$
The median age of 88 patients was similar with the previous reports [13]. In spite of the more women in our study, the critically ill group was still mainly constituted of old males, and the result may suggest the old males more easily developed to critically ill patients [14-17]. In particular, the analysis showed the age was positively correlated with some indexes, such as CRP, ferritin, LDH, IL-2R, IL-6, IL-8, IL-10 and TNF- $\alpha$, which may further elucidate that the severe inflammatory response more possibly take place among the old population. The BMI was also observed to decrease in critically ill group, although the $p$ value was not satisfactory which may be caused by the insufficient sample capacity. It needed more evidence to elucidate whether the relatively low BMI makes the disease more severe.

According to the reports at the initiate phage of COVID-19, $64(46.4 \%)$ had 1 or more comorbidities of 138 patients, and was similar with $46.59 \%$ in our study. Wang reported the rate $(72.2 \%)$ of comorbidity in the ICU patients [18], and more than half of the severe and critically ill patients (53.13\% and $77.78 \%)$ also had the comorbidity in our study, which all revealed that coexisting conditions would intensify the severity of COVID-19.

In consistent with recent studies [6,10,19-20], fever $(71.59 \%)$ and cough $(59.09 \%)$ were also the most common symptoms. But among these 88 patients, 22 $(25.00 \%)$ were suffered with diarrhea which was much more than the previous data $(3.80 \%)$. Given to the feature that there were no mild patients in our ward, we supposed whether gastrointestinal symptom was correlated with severity of COVID-19, or because the patient waited a long time (11 days) for hospital admission in outbreak phase and they took some medicines outside the hospital.

In accordance with the research published on January 24, 2020, Huang and his colleague found the cytokines and chemokines were elevated in the COVID-19 patients, and made the comparison between ICU and non-ICU patients, which revealed the higher IL-2, IL-7, IL-10, TNF- $a$, GCSF, IP10, MCP1, MCP1A levels in the ICU patients. Then they proposed that the cytokine storm was associated with disease severity, and indicated the cell response of 
T-helper-1 (Th1) and T-helper-2 (Th2) cells were both activated [10]. We also discovered there were higher levels and proportion of the elevation of LDH, IL-2R, IL-6, IL-8, IL-10 and TNF-a, furthermore, there were only higher levels of CRP and ferritin in the critically ill COVID-19 patients. By contrast, the identical phenomenon likewise occurred in both Severe Acute Respiratory Syndrome Coronavirus (SARS-CoV) and Middle East Respiratory Syndrome Coronavirus (MERS-CoV) infection [21,22], which was associated with pulmonary inflammation and extensive lung damage.

In our present study, there are still some notable limitations. Some cases had incomplete information of the exposure history, and the laboratory parameters in view of objective lack of infrastructure and subjective wishes of patients. And the statistical bias may influence our results because of the constricted sample capacity. There is another limitation also should be addressed is that children not been evaluated, which because children are not hospitalized in our ward. These would have been particularly useful to understand the difference of inflammatory cytokines between children and adults, since major studies reported that children have in general a milder disease with rare exception [23].

In conclusion, our results indicated that there were $29(32.95 \%)$ patients occurring liver damage, this may be caused by the direct attack of COVID-19 virus [24] or by use of drugs, which would affect the liver function [25]. Through the comparison between symptoms and inflammatory indexes, we found that dyspnea and fever were correlated with some indexes, such as lymphocyte count, CRP and inflammatory cytokines, which could suggest us to take measures in these patients with dyspnea or fever at the early stage of COVID-19 and that these patients symptom severity might be more severe than those without dyspnea or fever.

\section{Abbreviations}

COVID-19: novel coronavirus disease 2019; BMI: body mass index; CAD: coronary atherosclerotic heart disease; 2019-nCoV: 2019 novel coronavirus; SARS: severe acute respiratory syndrome; MERS: Middle East respiratory syndrome; ICU: intensive care unit; ESR: Erythrocyte sedimentation rate; CRP: C-reactive protein; CT: computed tomography; RT-PCR: real time polymerase chain reaction; ALT: alanine aminotransferase; AST: aspartate aminotransferase; IL: Interleukin; TNF-a: Tumor Necrosis Factor a; SD: standard deviation; IQR: interquartile range; DM: diabetes mellitus; COPD: chronic obstructive pulmonary disease; WBC: white blood cell count; PT: prothrombin time; Th1: T-helper-1; Th2: T-helper-2;
SARS-CoV: Severe Acute Respiratory Syndrome Coronavirus; MERS-CoV: Middle East Respiratory Syndrome Coronavirus.

\section{Acknowledgements}

This work was supported by the fund from Novel Coronavirus Pneumonia emergency research project of Shandong University (2020XGA02).

\section{Ethics approval and consent to participate}

Ethics Committee of Qilu Hospital of Shandong University approved this study.

\section{Competing Interests}

The authors have declared that no competing interest exists.

\section{References}

1. Lu H, Stratton CW, Tang YW. Outbreak of pneumonia of unknown etiology in Wuhan, China: The mystery and the miracle. J Med Virol. 2020; 92: 401-2.

2. Hui DS, E IA, Madani TA, et al. The continuing 2019-nCoV epidemic threat of novel coronaviruses to global health - The latest 2019 novel coronavirus outbreak in Wuhan, China. Int J Infect Dis. 2020; 91: 264-6.

3. [Internet] Wuhan Municipal Health Commission. Report of novel coronavirus-infected pneumonia in China. 2020 March. http://wjw.wuhan. gov.cn.

4. Paules CI, Marston HD, Fauci AS. Coronavirus Infections-More Than Just the Common Cold. JAMA. 2020; 323:707-8.

5. Wuhan Municipal Health Commission. Report of clustering pneumonia of unknown etiology in Wuhan City. 2019. http://wjw.wuhan.gov.cn.

6. Chen NS, Zhou M, Dong X, et al. Epidemiological and clinical characteristics of 99 cases of 2019 novel coronavirus pneumonia in Wuhan, China: a descriptive study. Lancet. 2020; 395: 507-13.

7. Zhu N, Zhang D, Wang W, et al. A Novel Coronavirus from Patients with Pneumonia in China, 2019. N Engl J Med. 2020; 382: 727-33.

8. Rodriguez-Morales AJ, Cardona-Ospina JA, Gutierrez-Ocampo E, et al. Clinical, laboratory and imaging features of COVID-19: A systematic review and meta-analysis. Travel Med Infect Dis. 2020; 34: 101623.

9. Novel Coronavirus Pneumonia Emergency Response Epidemiology Team. Vital surveillances: the epidemiological characteristics of an outbreak of 2019 novel coronavirus diseases (COVID-19)-China, 2020. http://weekly. chinacdc.cn.

10. Huang C, Wang Y, Li X, et al. Clinical features of patients infected with 2019 novel coronavirus in Wuhan, China. Lancet. 2020; 395: 497-506.

11. Wu Z, McGoogan JM. Characteristics of and Important Lessons from the Coronavirus Disease 2019 (COVID-19) Outbreak in China: Summary of a Report of 72314 Cases from the Chinese Center for Disease Control and Prevention. JAMA. 2020; 323:1239-42.

12. $\mathrm{Lu} \mathrm{H}, \mathrm{Ai}$ J, Shen $\mathrm{Y}$, et al. A descriptive study of the impact of diseases control and prevention on the epidemics dynamics and clinical features of SARS-CoV-2 outbreak in Shanghai, lessons learned for metropolis epidemics prevention. medRxiv. 2020. doi: https://doi.org/10.1101/2020.02.19. 20025031.

13. Chen NS, Zhou M, Dong X, et al. Epidemiological and clinical characteristics of 99 cases of 2019 novel coronavirus pneumonia in Wuhan, China: a descriptive study. Lancet. 2020; 395: 507-13.

14. Badawi A, Ryoo SG. Prevalence of comorbidities in the Middle East respiratory syndrome coronavirus (MERS-CoV): a systematic review and meta-analysis. Int J Infect Dis. 2016; 49: 129-33.

15. Channappanavar R, Fett C, Mack M, et al. Sex-Based Differences in Susceptibility to Severe Acute Respiratory Syndrome Coronavirus Infection. J Immunol. 2017; 198: 4046-53.

16. Jaillon S, Berthenet K, Garlanda C. Sexual Dimorphism in Innate Immunity. Clin Rev Allerg Immu. 2019; 56: 308-21.

17. Dryden M, Baguneid M, Eckmann C, et al. Pathophysiology and burden of infection in patients with diabetes mellitus and peripheral vascular disease: focus on skin and soft-tissue infections. Clin Microbiol Infec. 2015; 21 (Suppl 2): S27-S32.

18. Wang DW, Hu B, Hu C, et al. Clinical Characteristics of 138 Hospitalized Patients With 2019 Novel Coronavirus-Infected Pneumonia in Wuhan, China. Jama-J Am Med Assoc. 2020; 323: 1061-9.

19. Li Q, Guan XH, Wu P, et al. Early Transmission Dynamics in Wuhan, China, of Novel Coronavirus-Infected Pneumonia. New Engl J Med. 2020; 382: 1199-207.

20. Guan $\mathrm{W}, \mathrm{Ni} \mathrm{Z}, \mathrm{Hu} \mathrm{Y}$, et al. Clinical Characteristics of Coronavirus Disease 2019 in China. New Engl J Med. 2020; 382: 1708-20. 
21. Wong $\mathrm{CK}$, Lam CWK, Wu AKL, et al. Plasma inflammatory cytokines and chemokines in severe acute respiratory syndrome. Clin Exp Immunol. 2004; 136: 95-103.

22. Mahallawi WH, Khabour OF, Zhang Q, et al. MERS-CoV infection in humans is associated with a pro-inflammatory Th1 and Th17 cytokine profile. Cytokine. 2018; 104: 8-13.

23. Parri N, Lenge M, Buonsenso D, et al. Children with Covid-19 in Pediatric Emergency Departments in Italy. N Engl J Med. 2020; 383: 187-90.

24. Yeo C, Kaushal S, Yeo D. Enteric involvement of coronaviruses: is faecal-oral transmission of SARS-CoV-2 possible? Lancet Gastroenterol. 2020; 5: 335-7.

25. Zhang C, Shi L, Wang FS. Liver injury in COVID-19: management and challenges. Lancet Gastroenterol. 2020; 5: 428-30. 